\title{
Swift Nobel physics prize for superconductivity researchers
}

\section{London}

A YEAR of unprecedented excitement in solid-state physics has been capped by the award of this year's Nobel prize in physics to the two scientists who started it all off. J. Georg Bednorz, 37, and K. Alex Müller, 60, both of the IBM Zürich Research Laboratory in Rüschlikon, Switzerland, have been cited for "the discovery of superconductivity in ceramic materials". Their report, published just 13 months ago, of superconductivity at $35 \mathrm{~K}$ $\left(-238^{\circ} \mathrm{C}\right)$ in a mixed oxide of lanthanum, barium and copper triggered an explosion of research activity that has already led to superconductors that work at liquidnitrogen temperature, with the promise of room-temperature superconductors just around the corner.

Bednorz and Müller had been looking for superconductivity in mixed oxides since 1983. The superconductors with the highest transition temperatures $\left(T_{c} \mathrm{~s}\right)$ then known were alloys of metals, with the record standing at $23.3 \mathrm{~K}$ for $\mathrm{Nb}_{3} \mathrm{Ge}$. But this record had been set in 1973, and ten years of experimentation with other intermetallic alloys had failed to increase $T_{c}$. Müller, who had worked on insulating oxides in other contexts, focused instead on the known oxide superconductors, chief among which was a mixed oxide of barium, lead and bismuth, $\mathrm{BaPb}_{1-x} \mathrm{Bi}_{x} \mathrm{O}_{3}$, with a $T_{c}$ of $13 \mathrm{~K}$ and a crystal structure belonging to the class called perovskite.
Using crystal-chemical arguments to narrow the search, Müller decided to concentrate on perovskites containing nickel or copper, and enlisted the help of Bednorz, a skilled experimentalist. After two years without success, the breakthrough resulted from reading a paper by Bernard Raveau and colleagues, at the University of Caen, which described the synthesis of a lanthanum-barium-copper oxide perovskite with copper in a mixedvalence state. Bednorz and Müller set out to synthesize this compound, and here luck intervened. Bednorz and Müller's synthesis procedure was subtly different from that used by the Caen group, and led to a mixture of phases, instead of the pure phase they had been trying to make. One of the 'rogue' phases - also a mixedvalence oxide with a perovskite-related structure - turned out to be the $35-\mathrm{K}$ superconductor.

The events that followed are now well known: the confirmation of Bednorz and Müller's results by Shoji Tanaka's group in Tokyo, followed rapidly by groups at the University of Houston, Bell Laboratories, Bell Communications Research and the Chinese Academy of Sciences; the race to even higher $T_{c} \mathrm{~s}$, led by Paul Chu at Houston and collaborators, culminating in the discovery by Chu's group (and perhaps simultaneously by Z.X. Zhao and colleagues in China) of superconductivity at $90 \mathrm{~K}$ (above liquid-nitrogen temperature)

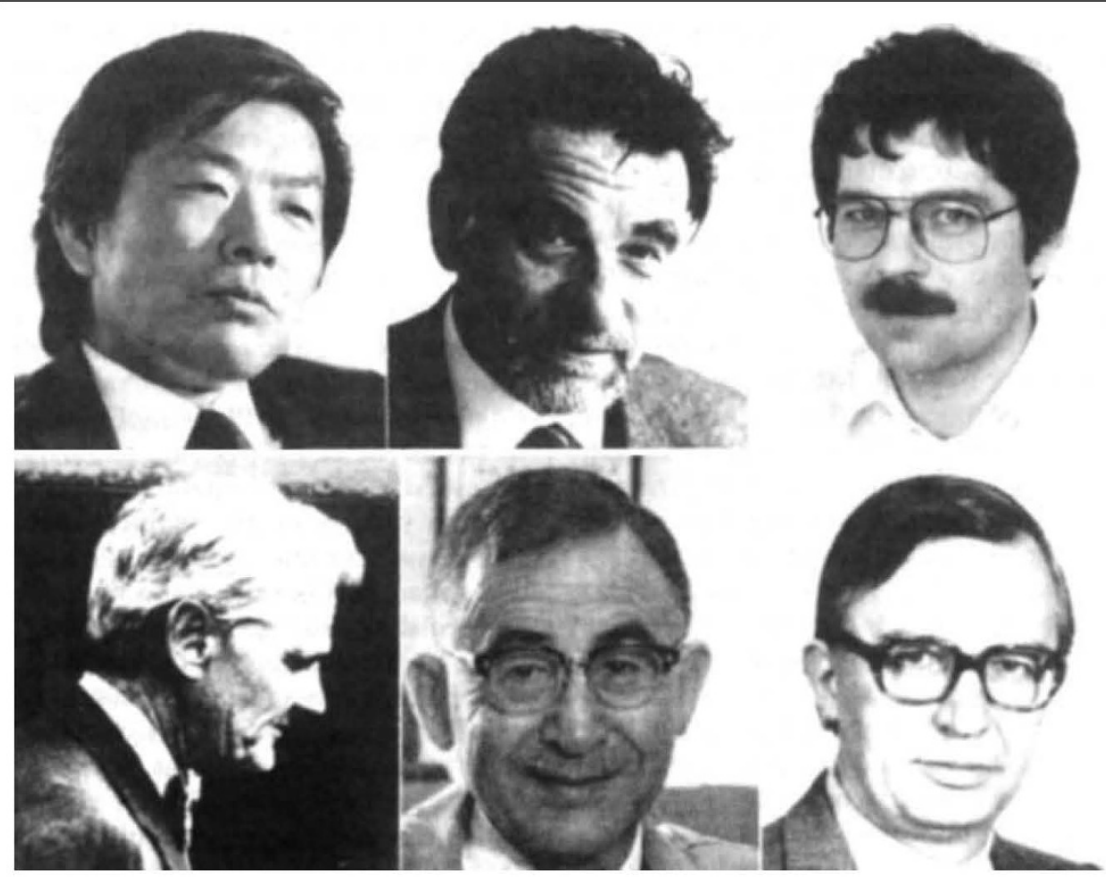

This year's line-up of Nobel laureates in the sciences. Top row: immunologist Susuma Tonegawa, winner of the prize in physiology/medicine (see Nature 329, 570; 1987), physicists Alex Müller and Georg Bednorz (see above). Bottom row: the chemists Donald J. Cram, Charles J. Pederson and Jean-Marie Lehn (see left).
An Abitur agreement Hamburg

AFTER more than two years of discussions, the ministers in charge of culture and education of the West German Länder (regions) have agreed on a uniform Abitur, the final examination after nine years in the gymnasium (secondary school). The crucial point is that from 1989 each $L a ̈ n d$ will accept the Abitur of another Länd. The Social Democratic model of KollegSchulen (in Nordrhein-Westfalen), which includes vocational education, was also accepted by Christian Democratic ministers. Everyone at a gymnasium must now study two of three subjects - German, a natural science (including mathematics) and one foreign language - through the Oberstufe and as an Abitur examination.

Jürgen Neffe

in the yttrium-barium-copper oxide system.

Chu had been widely tipped as a strong candidate for a share of the Nobel prize, and there are those in the field who feel that he deserved the honour. (One physicist pointed out that, owing to the time taken to publish their results, Bednorz and Müller had a nine-month start on Chu, and yet they did not find the $90-\mathrm{K}$ superconductor.) Others argue that Chu's breaking of the liquid-nitrogen barrier was more of a technological breakthrough than a scientific advance (it is not yet known whether the physics of the $90-\mathrm{K}$ superconductors differs from that of their $35-\mathrm{K}$ relations), and that its importance will depend on the rate of progress to still higher temperatures.

But the Nobel committee's choice may have been governed by more practical considerations: by giving the prize to Bednorz and Müller alone they avoided any controversy over priority between the Chinese and Houston groups; also, Chu's paper describing the $90-\mathrm{K}$ superconductor did not appear until more than a month after the 1 February deadline for Nobel nominations. Bednorz and Müller themselves are said to have just slipped in before the deadline, the result being a rare approach to fulfilment of the words of Alfred Nobel's will, which states that prizes shall be given to those who, "during the past year, shall have conferred the greatest benefit on mankind".

When the congratulations are passed round in Rüschlikon this week, some will certainly be due to the laboratory itself, for the second Nobel prize in as many years. Like last year's winners of the physics prize, Gerd Binnig and Heinrich Rohrer (inventors of the scanning tunnelling microscope), Alex Müller is an IBM Fellow. IBM's policy of allowing fellows to pursue projects of their own choosing for five-year periods thus appears to have paid off handsomely, at least in prestige. 